\title{
Tensile strength and corrosion resistance properties of porous Al2O3/Ni composites prepared with rice husk pore-forming agent
}

\begin{abstract}
The mechanical performance and chemical stability of porous alumina materials operating under harsh service conditions are of utmost importance in understanding their operational behavior if they are to stand the test of time. In the present study, the joint effect of nickel (Ni) reinforcement and rice husk $(\mathrm{RH})$ pore-forming agent (PFA) on the tensile strength and the corrosion resistance properties of composite porous alumina ceramics was studied. To exploit the potential of this new porous alumina system, plain and Ni-reinforced porous alumina samples (Al2O3-xNi-RH; $x=2,4,6$ and $8 \mathrm{wt} \%$ ) were developed through the powder metallurgy technique. Comprehensive investigation on the tensile strength properties of the developed porous alumina ceramics showed that relative to the plain sample (tensile strength and elastic modulus; 6.1 MPa and $1201 \mathrm{MPa}$ ), the presence of highly stable Ni3A12SiO8 spinelloid promoted the tensile strength enhancement (12.6-6.4 MPa) and the elastic modulus decline (897-627 MPa) of the composite samples. Similarly, corrosion resistance test was performed on the composite porous alumina samples in both $10 \mathrm{wt} \% \mathrm{NaOH}$ and $20 \mathrm{wt} \%$ $\mathrm{H} 2 \mathrm{SO} 4$ hot aqueous solutions. Overall, the composite samples demonstrated superior chemical stability in $\mathrm{NaOH}$ solution as compared with the plain sample. On the other hand, the composites were more prone to attack in $\mathrm{H} 2 \mathrm{SO} 4$ solution, except for the Al2O3-2Ni-10RH composite sample which maintained its superiority over the plain counterpart.
\end{abstract}

Keyword: Porous alumina; Composites; Rice husk; Mechanical properties; Corrosion resistance 